\title{
Testimonio de un saber sobre la educación: tres periódicos pedagógicos decimonónicos
}

\author{
Testimonies of Educational Knowledge: \\ Three Nineteenth-century Pedagogical Newspapers
}

\author{
Juan Pedro Galván Gómez \\ Facultad de Filosofía y Letras, UNAM \\ axiomal@prodigy.net.mx
}

\begin{abstract}
Resumen
La incursión en la prensa de maestros y personajes interesados en la educación, a lo largo del último tercio del siglo XIX, dio nacimiento a un tipo de prensa que podemos denominar como pedagógica.

A través del estudio de la prensa decimonónica, en especial de la pedagógica, es posible develar los elementos del discurso que, con el tiempo, se convirtieron en un saber sobre la educación, es decir, la pedagogía.

La manera de aproximarnos a la lógica del pensamiento educativo de la época se basa, principalmente, en el análisis del discurso sobre un ideal de transmisión del conocimiento en el salón de clase, en la organización escolar y en la necesidad de formar un maestro capaz de conocer y manejar estos conocimientos.
\end{abstract}

Palabras clave: Prensa siglo XIX, prensa pedagógica, pedagogía.

\begin{abstract}
The involvement of teachers and persons with interests in education in the press throughout the last third of the nineteenth-century gave birth to a type of periodical publications that can be denominated pedagogical. Through the study of the nineteenth-century press, especially the pedagogical press, it is possible to reveal elements that over time constituted a body of knowledge of education, identified as pedagogy. Our approach to studying the logic of educational ideology of the time is based on discourse analysis of texts on the ideal of knowledge transmission in the classroom, on school organization, and on the need to train teachers capable of understanding and handling this knowledge.
\end{abstract}

Abstract

Key words: pedagogical press, pedagogy, Mexico, nineteenth century, discourse analysis 


\section{Introducción}

Con el nacimiento del Estado Mexicano, en la segunda década del siglo XIX, se inició una ardua y accidentada labor para conformar las estructuras económica, política y social que el país requería. Uno de esos trabajos fue, por supuesto, la organización de la educación.

Primero se pensó que para educar al pueblo lo ideal sería abrir la mayor cantidad de escuelas, visión que prevaleció por mucho tiempo. Fue necesario esperar cinco décadas para que la idea de difundir la educación se ampliara con la consideración de que también era indispensable formar personas capaces de enseñar con base en una doctrina pedagógica y con conocimientos de organización escolar.

En tanto se llegaba a ese punto, la organización de la educación durante la primera mitad del siglo XIX se dio, principalmente, a través de diversos ensayos legislativos que pretendieron dar una estructura conveniente a la enseñanza en todos sus niveles, recurriendo reiteradamente a la intención de abrir más escuelas. Sin embargo, las formas de transmisión del conocimiento siguieron siendo aquellas basadas en el memorismo.

Hubo que esperar hasta el último tercio del siglo XIX para que se diera una reflexión en torno a la apremiante necesidad de aplicar una metodología en la enseñanza. Mas no sólo bastaba adoptar un método, también era fundamental su difusión, la cual fue posible gracias a la iniciativa tanto de personajes preocupados por el estado de la educación, como por maestros que vieron en la prensa el medio para dar a conocer sus ideales educativos.

Por ello resulta de especial relevancia que el estudioso de la historia de la educación fije su mirada en este tipo de fuentes que posibilitan un acercamiento al pensamiento pedagógico de un lapso determinado en el que las publicaciones educativas son actores de primera mano al ser producto de la pluma de los mismos promotores de las reformas educativas es decir: los maestros.

En general, el devenir del periodismo a lo largo del siglo XIX tuvo como característica la polémica y la confrontación entre las facciones en lucha por el poder político. No obstante, en la época conocida como la República Restaurada, el pensamiento intelectual tuvo un lapso de auge, pues los escritores enfocaron su atención hacia otros tópicos más allá del político.

Con Juárez en la presidencia "el renacimiento intelectual de México encontró un amplio campo de acción dentro de un régimen de libertad de expresión y de prensa"(Lepidus, citado en Lombardo, 1992: 19). Hacia 1868, este florecimiento cultural se pretendió alentar cuando Pedro Santacilia, redactor del Diario Oficial y yerno del presidente, publicó un trabajo titulado: Del movimiento literario en México (Lombardo, 1992: 19). En éste, "Santacilia pretendió adjudicar el renacimiento de las letras a la restauración de la política juarista" 
(Batiz, 1963, citado en Lombardo, 1992: 19) y consideraba que, como la gente se había cansado del debate político en la prensa, era necesario escribir textos sobre historia, libros para la enseñanza, obras de ciencia, novelas, poesías, dramas, manuales, mapas.

Lo anterior era obligado, pues para entonces se venían presentando numerosos avances de diverso tipo que era importante dar a conocer entre los que Santacilia mencionaba a la Academia Industrial, la Lonja Mercantil, los clubes políticos, la Sociedad Filarmónica, la empresa del ferrocarril, la fábrica de gas, la de resina para el alumbrado, el telégrafo, los colegios, liceos, y numerosas veladas literarias (Batis, citado en Lombardo, 1992: 20). Esta invitación al trabajo intelectual, surgida de un colaborador del presidente, pudo tener el propósito de desviar la atención sobre la actividad de Juárez y sus facultades extraordinarias para legislar, asimismo, es un elemento que pudiera explicar, pero no el único, la multiplicación de la denominada prensa especializada. Empero, ésta no debe considerarse como producto de la voluntad de una persona, sino como el resultado de diversas inquietudes sociales enmarcadas por un entorno de cambios políticos que dieron nacimiento al Estado moderno mexicano, inquietudes que encontraron expresión en las publicaciones periódicas. Por tanto, es posible observar "que a los diversos acontecimientos sociales correspondieron formas de informar que abrieron paso a distintos tipos de periódicos" (Lombardo, 1992: 12).

\section{La prensa especializada}

Los periodos de la reforma y el porfirismo fueron etapas de cambios que, a su vez, provocaron inquietud social y que conformaron el escenario en el cual se ubica la prensa especializada. Es en esta segunda mitad del siglo XIX en la que el periódico, como reacción a nuevas necesidades sociales, experimenta innovaciones tanto en la forma como en el contenido; el reportaje y la entrevista aparecen como géneros periodísticos, lo que llevaría a hacer de estas publicaciones recipientes de discursos diversos y, por tal motivo, habría nuevos lectores destinatarios con perfiles e intereses diversos.

Al diversificarse la temática periodística, se nos presenta un espectro de distintas publicaciones que diferentes estudiosos del tema dividen con mayor o menor detalle. Por ejemplo, en El estadio. La prensa en México, Abramo y Barberena (1998: 14-18) las clasifican de la siguiente forma: la primera en prensa obrera y artesana; la segunda en prensa orgánica, que a su vez subdivide en política, religiosa, oficial, científica y técnica y comercial; en una tercera categoría ubica a la prensa noticiosa, dejando al final a la que califica como de géneros menores, la que divide en literaria y teatral, femenil, infantil y juvenil y mixta. Por lo que toca a la prensa infantil y juvenil, señala que hubo un reducido número de títulos dedicados a este segmento de la población, en las que se editaban cuentos y se incluían casi siempre cuestio- 
nes de orden moral y/o religioso. Algunos de estos "organismos", como los denominan estos autores, estuvieron vinculados a corrientes pedagógicas (Abramo y Barberena, 1998). La observación que hacen estos investigadores constituye un pequeño acercamiento que, aunque no ahonda demasiado en este tipo de impresos, sí nos hace poner nuestra atención en ellos.

Por su parte, Ruiz Castañeda (1984) hace una división un poco más amplia sobre los impresos especializados. En esta propuesta encontramos publicaciones agropecuarias, artísticas, científicas, educativas, sobre espectáculos y recreación, femeninas, de industria y comercio, literarias, obreras y religiosas. Según la misma autora, algunas de las publicaciones educativas estaban destinadas a los niños y otras a los maestros, las últimas servían para difundir los avances de la pedagogía e intentaron cohesionar al magisterio.

Otra clasificación interesante es la que hace Florence Toussaint, quien divide la prensa en dos grandes grupos: las publicaciones políticas y las especializadas. A su juicio, el primero obedece tanto a la toma de partido por algunos políticos o en favor de actos de gobierno, y, en otros casos, al mejoramiento o defensa de estratos específicos: obreros o mujeres. En este rubro ubica a los periódicos político-literarios, a los religiosos, a los humorísticos (con o sin caricaturas), a los informativos, a los obreros, a los oficiales y a los femeninos.

El segundo grupo lo divide por los objetos que constituyen sus respectivas materias de reflexión o noticia (Toussaint, 1989). Así, las publicaciones especializadas abarcarían las agrícolas, las científicas, las médicas, las de comercio, las de economía, minería e industria; las de literatura y las educativas, infantiles y de espectáculos.

El hecho de que ubique en una misma clasificación a las publicaciones educativas e infantiles, hace suponer que no quiso marcar una tajante diferencia entre ambas, tal vez por no ser de su inmediato interés hacer este tipo de análisis o para dejar que quienes quisieran profundizar en el tema, lo hicieran, pues es obvio que Toussaint maneja las diferencias entre ambas al señalar motivaciones distintas entre las ediciones pedagógicas y las infantiles. Afirma que las primeras contribuyeron a la educación y a la reflexión pedagógica y metodológica, y las segundas fueron herramientas que cubrieron las necesidades de educación extraescolar y de entretenimiento cultural de los lectores (Toussaint, 1989).

El contrastar las anteriores clasificaciones de las publicaciones periódicas no tiene la finalidad de adoptar una o sintetizarlas y proponer otra más, que tendría sólo ligeras diferencias. Creo más bien que lo que debe despertar nuestro interés es la diversidad temática que nos presentan y sus posibles diferencias y especificidades, lo que las convierte en fuente importante para su estudio, ya sea de manera general o particular.

Esa amplia gama de tópicos que nos muestran sus contenidos nos descubre un panorama en el que se percibe el desarrollo de una sociedad que se fue haciendo más compleja a lo largo del último tercio del siglo XIX. La prensa, además de continuar debatiendo sobre política, fue el medio a través del cual se expusieron las variaciones en las pautas de conduc- 
ta de una sociedad que era reconfigurada por los cambios políticos y económicos, fruto de una, hasta entonces, desconocida y relativa estabilidad política.

La reflexión y el pensamiento que las nuevas relaciones sociales produjeron, tuvieron en la prensa, sus mismos actores lo aseguraban, el elemento propagador de sus ideas. Laureana Wright, por ejemplo, opinaba que una expresión del adelanto de la civilización consistía "en la fundación de un periódico femenil destinado a sostener los intereses sociales, los derechos y las prerrogativas sociales de nuestras compatriotas." (Las Hijas del Anáhuac, diciembre de 1887: 2).

La llegada de la doctrina socialista a México a mediados del siglo XIX dio origen a una tipología periodística denominada obrera, en la que es posible observar que la toma de conciencia de los trabajadores sobre la situación de cambio en las relaciones de trabajo los llevó a agruparse en sociedades mutualistas o cooperativas desde donde luchar para tener participación en la vida política y obtener una educación que les permitiera mejorar sus condiciones de vida; así, si la estrategia de lucha implicaba la agrupación y la instrucción, éstas deberían ser apoyadas por la comunicación, por lo que recurrieron al periodismo (Ruiz et al., 1957).

El hecho que impulsó la creación de escritos de carácter obrero fue sin duda el desarrollo del capitalismo, ante el cual los artesanos crearon asociaciones de tipo mutualista, antecedentes de la organización sindical, las que a principios de los años setenta del siglo XIX se aglutinaron en el "Gran Círculo de Obreros de México", considerada la primera gran central de trabajadores de la República (Bringas y Mascareño, 1988). La publicación oficial del Círculo fue el periódico El Socialista. Dedicado a defender los derechos e intereses de la clase trabajadora de 1871.

Otro representante más de la prensa especializada fueron los periódicos femeninos. Acorde con los cambios de fin de siglo, este tipo de periodismo muestra las inquietudes propias de las mujeres de la época, que van desde su emancipación a través de la educación, de la necesidad de ampliar su campo de actividades, hasta problemas propiamente políticos (Ruiz et al., 1957). Para María del Carmen Ruiz Castañeda (1957), el género inicia con el título Las Hijas del Anáhuac, editado entre 1873 y 1874, cuya directora fue la señora Concepción García y Ontiveros, más ello no significa que la mujer no estuviera presente con anterioridad en el devenir de las publicaciones periódicas mexicanas, pues se le puede encontrar como impresora, colaboradora o lectora de algún periódico o revista de la primera mitad del siglo XIX. Al respecto, Alicia Pereda (2003) afirma que en los primeros treinta años de dicha época El Diario de México y El Iris identifican a las mujeres como destinatarias y lectoras potenciales.

La importancia de este tipo de publicaciones radica en el hecho de que las opciones educativas para las mujeres fueron pocas y sólo alcanzaron a un reducido número de ellas, por lo que los periódicos y revistas cubrieron una importante función educativa en el México de principios del siglo XIx (Becerril, 2000; Alvarado, 2004). Ello amplió las posibilidades 
educativas para el "bello sexo" y fue entonces que se abrió, aunque muy lentamente, el mundo cerrado de las novohispanas (Becerril, 2000).

Además de Las Hijas del Anáhuac, existieron títulos como El Correo de las Señoras de 1883, El Álbum de la Mujer de 1883 y Las Violetas del Anáhuac de 1887 a 1889. En éste último localizamos los nombres de dos colaboradoras: Dolores Correa Zapata y Matiana Murguía, mujeres que reflejan la evolución social de finales del siglo XIX y que dejan testimonio de ello en su participación editorial y educativa. La primera sería, hacia 1890, subdirectora de la primaria anexa a la Normal y, por su parte, Matiana Murguía fue directora de la Escuela de Párvulos, anexa también a la Normal del Distrito Federal. Ambas encontraron en el periodismo espacio para dar a conocer sus ideas sobre la educación femenina y sobre la discriminación de que eran objeto las maestras con respecto a sus colegas del género opuesto, lo que se comprueba, entre otras cosas, en la diferencia de salario que percibían.

No resistimos la tentación de al menos mencionar otras publicaciones, de tipo religioso, que nos permiten ver cómo la religión católica iría perdiendo el monopolio de la fe en México. De ello son testimonio publicaciones periódicas como El Faro, que fue el órgano de la Iglesia presbiteriana en México, el cual elogia la separación de la Iglesia y el Estado y la libertad de cultos. Este impreso, dice Ruiz Castañeda (1984), responsabilizaba al clero católico de todo los males que sufría el país, y lo acusaba de impedir el desarrollo intelectual y de llenar a la República de fanáticos e ignorantes.

Otro género que comienza a esbozarse a fines del siglo decimonono es el de las revistas masculinas que, a decir de Consolación Salas (1998), forma parte de la industria del ocio y cuyo ámbito de desarrollo se encuentra fuera de lo doméstico y rutinario, ya que se les rodea de un halo de glamour y mundanería y manejan una línea editorial en donde predomina el optimismo y una visión positiva de la vida. Entre los títulos que abarcan esta temática podemos enunciar los siguientes: Frégoli de 1897, El Cómico de 1896, El Burro de 1900, Frivolidades de 1910, entre otras.

\section{La prensa pedagógica}

En el campo de las publicaciones con temas específicos encontramos también a la denominada prensa pedagógica, la cual tiene como iniciador, a decir de varios autores (Larroyo, 1973; Meneses, 1998; Castellanos, 1909), la figura de Antonio P. Castilla quien en 1871 publicó el periódico La Voz de la Instrucción, o sea el libro primero del maestro. Semanario destinado al progreso de la enseñanza y a la defensa de los intereses materiales y morales del profesorado mexicano, "desde donde dio las primeras lecciones de pedagogía" (Castellanos, 1909: 39).

Esta publicación aborda la situación de la enseñanza de la época y nos ofrece un panorama del estado de la educación, tanto en lo que se refiere a los sistemas utilizados al momento 
de publicarse, como a la falta de preparación del profesorado, lamentando que difícilmente se podían encontrar en los pueblos maestros que supieran leer bien, escribir y contar, y que en lo económico, salvo pocas excepciones, su situación era de indigencia (Castilla, 1871: 1).

El apoyo que debía prestar la prensa al desarrollo y difusión de las ideas educativas se expresa constantemente; Manuel Cervantes afirmaba que "la necesidad de una gran reforma en la instrucción primaria nos trae al terreno de la prensa, donde podremos buscar con ahínco y buena fe el remedio de aquellos males que tanto nos han hecho padecer" (Cervantes, 1874: 1).

A través de su publicación, el profesor Castilla nos llama la atención sobre los elementos que él consideraba como necesarios para el perfeccionamiento de la instrucción: la prensa dedicada a la enseñanza, las escuelas normales y la inspección facultativa, este último el más importante desde su punto de vista. En este aspecto coincidimos con el maestro Castilla, pues considerar a la prensa como uno de los factores necesarios para la mejoría de la instrucción fue consecuencia de las expectativas que estas publicaciones crearon para difundir un saber sobre la enseñanza, los modelos conocidos de organización escolar y por dar a conocer la forma de transmitir el conocimiento en el salón de clase. Todo ello, por supuesto, dirigido a los maestros en funciones, en formación o para todo aquel que tuviera bajo su responsabilidad un establecimiento educativo.

Ahora bien, considerar a un segmento de la producción periodística de fines del siglo XIX como prensa pedagógica se debe a que en estas publicaciones encontramos la exposición de esta práctica y su respectivo discurso; y a pesar de que el vocablo que la califica era conocido desde la antigüedad griega, su percepción y cientificidad era innovadora, aún más en nuestro país, donde se iba generalizando la discusión pública sobre dichas cuestiones.

Abraham Castellanos y Antonio P. Castilla coinciden en que la prensa era importante en la difusión de las ideas educativas; aunque para el primero ésta, junto al poder público, los filósofos y los maestros, fueron factores fundamentales del proceso que otorgó un estatus científico a la pedagogía y cuya relación impulsó la conformación de un programa teórico, el concepto de método pedagógico y la disciplina en general (Castellanos, 1909).

En este orden de ideas podríamos concluir que el discurso educativo de este tipo de prensa se conforma como el elemento que sustentó y difundió la naciente práctica pedagógica, iniciada a partir de la acción legislativa del poder público a su vez generada por el debate de los filósofos (maestros, políticos, periodistas y todos los interesados en el tema), en torno de la estructuración de un sistema educativo. Para dicho objeto, resultaba vital que el maestro se formara dentro de una metodología pedagógica acorde a la concepción educativa que se tenía para entonces en los países más desarrollados y con ello se convirtiera en el agente multiplicador de la nueva disciplina.

Ezequiel A. Chávez también ofrece una visión sobre el papel que ocuparon los periódicos de tema escolar dentro del proceso educativo del siglo XIX. Para él la capital fue el foco de 
irradiación de los avances educativos y, desde su punto de vista, contó con tres manifestaciones que tuvieron efecto multiplicador en los adelantos escolares en el país. La primera fue la propagación de escuelas, al menos planteada por la legislación, que pretendía organizar y reorganizar la educación; la segunda consistía en la realización de reuniones científicas, como los congresos educativos, en los que los interesados en el tema debatieron sobre el mejor sistema y método de enseñanza, nutriendo con sus planteamientos la legislación sobre la materia. Finalmente, considera la edición de publicaciones escolares como el medio que permitió la difusión de las nuevas ideas (citado en Meneses, 1998). Las ideas pedagógicas, en contraste con la opinión de Chávez, no solamente es posible ubicarlas en la capital de México, indicio de ello es la profusión de títulos de periódicos pedagógicos que podemos ubicar a lo largo del siglo XIX en distintos estados del país (véase cuadro 1).

La necesidad de reestructurar el sistema educativo con un andamiaje teórico proveniente de educadores como Froebel, Rébsamen, Laubscher o Carrillo, entre otros, da como uno de sus principales resultados el nacimiento de ediciones que reflejaron la preocupación por cambiar el estado de cosas. De ello nos percatamos en los objetivos que las publicaciones pedagógicas plantean en sus ediciones iniciales; como ejemplo podemos citar lo que al respecto publica $\mathrm{La}$ Enseñanza Moderna al afirmar que su tarea la realizaría en el ámbito de la instrucción pública, pues su pretensión sería dar a conocer los adelantos que se iban realizando en este campo y procuraría "demostrar los defectos de que adolecen algunos métodos de enseñanza adoptados en varios puntos del país" (La Enseñanza Moderna, octubre de 1897: 1).

Las ideas educativas, tanto internas como externas, tuvieron cabida en las publicaciones pedagógicas como se expresa en el primer número de La Enseñanza Objetiva, impreso que informaba a sus posibles lectores que: "no solo publicaremos métodos de autores extrangeros [sic] que vendrán en auxilio de los maestros, sino también las observaciones de los Sres. Profesores, que de algún tiempo a esta parte, se han ocupado en México de propagar prácticamente la enseñanza objetiva." (La Enseñanza Objetiva, enero de 1879: 1).

Como podemos ver estos impresos tocaban el tema del método de enseñanza, pero no sólo eso, también podemos conocer que alrededor de ellos se conformaron grupos interesados en el avance educativo, esto último no fue exclusivo de este género, Luz Elena Galván también lo ha observado en el estudio de la prensa infantil (Galván, 2004). Es el caso del Boletín Bibliográfico y Escolar (1891: 138), que sería testigo de la constitución de la Sociedad Pedagógica, a la vez que abrió sus páginas para que los miembros de esta agrupación expresaran sus ideas en torno a la pedagogía.

En El Porvenir de la Niñez (1870), se vislumbra otro tema abrigado en este tipo de publicaciones; el incipiente intento por cohesionar al gremio magisterial; uno de los indicios para esta afirmación, proviene de la reproducción en la edición del 16 de diciembre de 1870 del "Reglamento interior de la Asociación de Profesores de Primeras Letras en Zacatecas", cuyo 


\section{Cuadro 1}

\section{Lista de periódicos pedagógicos en México, siglo XIX}

\begin{tabular}{|c|c|c|}
\hline Título & Lugar de edición & Fecha de aparición \\
\hline El Mosaico Mexicano & México & 1836 \\
\hline El Diario de los Niños & México & 1839 \\
\hline El Museo Mexicano & México & 1843 \\
\hline La Esperanza & Campeche & 1863 \\
\hline El Porvenir de la Niñez & México & 1870 \\
\hline La Siempreviva & Mérida, Yuc. & {$[1870]$} \\
\hline La Luz & Durango & 1870 \\
\hline El Obrero del Porvenir & México & 1870 \\
\hline El Sábado & $\begin{array}{c}\text { San José de Iturbide, } \\
\text { Gto. }\end{array}$ & 1870 \\
\hline El Inspector de Instrucción Primaria & Zacatecas & 1871 \\
\hline La Enseñanza & Nueva York y México & 1870 \\
\hline La Escuela de las Primeras Letras & Guanajuato & 1870 \\
\hline El Instructor de los Niños & Coatepec, Ver. & 1870 \\
\hline La Educación & Guanajuato & 1871 \\
\hline La Voz de la Instrucción & México & 1871 \\
\hline El Hogar & Toluca & {$[1872]$} \\
\hline El Escolar & México & {$[1872]$} \\
\hline El Protector de la Infancia & Guadalajara, Jal. & 1872 \\
\hline El Correo de los Niños & México & 1872 \\
\hline La Edad Feliz & México & 1873 \\
\hline El Amigo de los Niños & Acapulco, Gro. & {$[1874]$} \\
\hline El Educador Mexicano & México & 1874 \\
\hline El Estudio & Puebla & 1875 \\
\hline La Enseñanza Objetiva & México & 1879 \\
\hline Álbum Recreativo & Mérida, Yuc. & 1882 \\
\hline El Instructor & Aguascalientes & 1883 \\
\hline El Instructor & Coatepec, Ver. & 1883 \\
\hline La Reforma de la Escuela Elemental & Coatepec, Ver. & 1885 \\
\hline
\end{tabular}




\begin{tabular}{|c|c|c|}
\hline Título & Lugar de edición & Fecha de aparición \\
\hline La Escuela Primaria & Mérida, Yuc. & 1886 \\
\hline Revista de la Instrucción Pública Mexicana & México & 1886 \\
\hline El Educador Práctico Ilustrado & $s / 1$ & 1886 \\
\hline La Escuela Primaria & Mérida, Yuc. & 1887 \\
\hline El Escolar Mexicano & México & 1888 \\
\hline México Intelectual & Jalapa, Ver. & 1889 \\
\hline La Escuela Moderna & México & 1889 \\
\hline Boletín Bibliográfico Escolar & México & 1891 \\
\hline La Bandera Escolar & Oaxaca & 1892 \\
\hline El Boletín de Instrucción Primaria y Normal & Oaxaca & 1892 \\
\hline El Escolar Zacateco & Zacatlán, Pue. & 1892 \\
\hline La Instrucción Pública & Sonora & 1892 \\
\hline Revista Pedagógica & Monterrey & 1892 \\
\hline La Sombra de Carrillo & Guanajuato & 1892 \\
\hline La Revista Escolar & Tabasco & 1892 \\
\hline El Eco Pedagógico & México & 1893 \\
\hline El Boletín Pedagógico & Toluca & 1894 \\
\hline Boletín de Instrucción Primaria & Nuevo León & 1894 \\
\hline El Niño Mexicano & México & 1895 \\
\hline La Educación Contemporánea & Colima & 1895 \\
\hline El Progreso Escolar & Guadalajara, Jal. & 1896 \\
\hline La Unión Escolar & Oaxaca & 1897 \\
\hline La Enseñanza Moderna & México & 1897 \\
\hline La Evolución Escolar & México & [1898] \\
\hline La Enseñanza Primaria & México & 1901 \\
\hline La Primaria & $s / 1$ & 1901 \\
\hline La Educación Nacional & Chihuahua & 1902 \\
\hline Boletín de la Instrucción Pública & México & 1903 \\
\hline La Enseñanza Normal & México & 1904 \\
\hline
\end{tabular}




\begin{tabular}{lcc}
\hline Título & Lugar de edición & Fecha de aparición \\
La Escuela Mexicana & México & 1904 \\
El Magisterio Nacional & México & 1904 \\
\hline
\end{tabular}

FUENTE: Elaboración propia con base en la consulta de periódicos de la época.

fin fue el de uniformar el método de enseñanza, su perfeccionamiento y difusión a través de la entidad. El reglamento nos hace suponer la existencia de una agrupación de profesores con un fin común y con la idea de ser reconocidos como profesionales de la educación.

Además de los señalados, otros títulos de publicaciones pedagógicas son: el Álbum Recreativo de 1882, el Boletín Bibliográfico Escolar de 1891, el Boletín de la Instrucción Pública de 1903, El Eco Pedagógico de 1893, La Educación Contemporánea de 1895, El Educador Práctico llustrado de 1886, La Enseñanza Moderna de 1897, La Escuela Primaria de 1886, La Evolución Escolar de 1899, La Reforma de la Escuela Elemental de 1885, La Unión Escolar de 1897, La Voz de la Instrucción de 1871, México Intelectual de 1889 y La Enseñanza Primaria de 1901, entre otros muchos.

Los citados impresos fueron espacios en donde se perciben las mutaciones culturales (expresión acuñada por F. Xavier Guerra) que se experimentaban en el último tercio del siglo XIX, muestra de ello es la aparición de una prensa que reflejó las inquietudes sociales de la época. Así, estas publicaciones fueron voz y presencia de cambios y de una clara tendencia hacia una mayor complejidad de la sociedad de la segunda mitad del siglo XIX.

Cabe aclarar que para que estas transformaciones afloraran tuvieron como marco un tiempo de cierta estabilidad política, como el que se presentó con la restauración de la República durante la administración de Juárez y Lerdo de Tejada, y con la encabezada posteriormente por Porfirio Díaz.

\section{Tres títulos representativos}

Transitar a través de la prensa pedagógica mexicana de finales del siglo XIX resulta una tarea ardua, no obstante, la elección de La Voz de la Instrucción de 1871, México Intelectual de 18891890 y La Enseñanza Primaria de 1901, son una muestra de ese vasto universo que nos permite reconocer el discurso que fue conformando las ideas básicas sobre una emergente práctica pedagógica en aquel contexto.

El criterio de elección de tales publicaciones en los años expresados líneas arriba, estriba en que la primera, al ser considerada como la iniciadora de una reflexión sobre la educación, nos ubica en los umbrales de una reforma educativa; la segunda, al aparecer en el periodo en 
el que se ubica el mayor debate en torno al perfeccionamiento de la enseñanza, nos da luz sobre el camino que seguiría el sistema escolar a finales del siglo XIX y principios del XX. Finalmente, el tercer impreso lo podemos considerar como el resultado de la aplicación práctica de las reflexiones anteriores a su aparición, toda vez que es el fruto de la iniciativa de un grupo de profesores de la Normal de la ciudad de México, quienes resultaron ser los receptores, tanto de las ideas educativas de la época, como del ideal de formación magisterial en escuelas especiales para ello.

\section{La Voz de la Instrucción}

En 1871 el profesor Antonio P. Castilla editó un periódico con el título de La Voz de la Instrucción, el cual iba seguido de un largo subtítulo que dejaba entrever sus propósitos: "O sea el libro primero del maestro. Semanario destinado al progreso de la enseñanza y a la defensa de los intereses materiales y morales del profesorado mexicano". La denominación de "libro primero" nos hace pensar que consideró a la obra como básica para la necesaria y urgente formación de los mentores, cuya consecuencia se reflejaría en el progreso general de la enseñanza. De igual manera, podemos inferir que al invocar la defensa de los intereses materiales y morales de los profesores, pone a descubierto la pobre percepción que la sociedad tenía de esta actividad; para revertir tal opinión se propuso la unificación del profesorado pues concluía que "el triste estado que guarda la profesión de maestro en la República, pide de voz en grito, un remedio eficaz; reclama la unión del profesorado todo, para reivindicar sus lastimados derechos" (Castilla, 1871: 65).

El impreso, en resguardo de la Hemeroteca Nacional de México, constó de 24 números en un pequeño formato de 21 x $14 \mathrm{~cm}$ con un total de 363 páginas y cuyo costo fue de un real en la capital y real y medio fuera de ésta. Los datos sobre el tiraje no se consignan en ninguna parte del impreso, sin embargo, su alcance geográfico fue amplio atendiendo a los remitidos de suscripciones y al intercambio de correspondencia que se señalan en sus páginas. Así, lo ubicamos en Córdoba, Veracruz, Jalapa, Puebla, Celaya, Cuernavaca, Guanajuato, Querétaro, Aguascalientes, Mazatlán, Río Verde, Fresnillo, Tlaxcala, San Luis Potosí, entre otras ciudades (Castilla, 1871: 11, 21, 34, 64).

${ }^{1}$ El 28 de diciembre de 1870 La lberia anunció que el profesor Antonio P. Castilla llegaría a radicar en el Distrito Federal. Este educador se estableció en la calle de la Cerca de Santo Domingo 16 con la Imprenta y Librería de la Enciclopedia de Instrucción Primaria que tenía en San Luis Potosí y en donde también dirigió un colegio de niños. En el local pondría a la venta obras propias, publicaciones nacionales y extranjeras en comisión, depósito en comisión de objetos útiles para la enseñanza, proporcionaría información sobre colegios y publicaría un semanario. En el año de 1871 Castilla editó un periódico con el título de La Voz de la Instrucción. Abraham Castellanos consideró que el profesor Castilla, por medio de su periódico, fue el que dio las primeras lecciones de pedagogía al profesorado mexicano, difundiendo las ideas más avanzadas de la pedagogía europea en didáctica y organización escolar. 
En la portada se indica que el fundador, redactor y editor propietario del periódico fue el propio Castilla, por lo que deducimos que el trabajo editorial de esta publicación fue producto del esfuerzo personal por presentar el estado de la situación escolar hasta 1871, de sintetizar los sistemas y métodos de enseñanza entonces utilizados y conocidos y de exponerlos de manera ordenada y sistemática con la intención de que fueran aplicados adecuada y eficientemente en los heterogéneos establecimientos educativos de la época. Esta tarea debería contribuir a la organización del trabajo escolar, pues el editor consideró que al dar a conocer los sistemas y métodos de enseñanza ofrecía la información necesaria para conocer y decidir cuáles serían los más adecuados de acuerdo con las características de cada establecimiento. Esto también permitiría proveer de cierta unidad a la enseñanza, en la cual se debería enfatizar la inspección facultativa verificada por profesores encanecidos en la carrera de la enseñanza (Castilla, 1871), y que para el profesor Castilla era el elemento más importante para hacer efectiva la educación en las escuelas. Consecuentemente, pensaba que si esta visión era adoptada, compartida y practicada por los profesores como parte de su formación, se estaría en camino hacia una revaloración del maestro.

En el semanario colaboraron Ignacio Manuel Altamirano e Ignacio Ramírez (Castilla, 1871: 17, 23), sin embargo, no fue posible distinguir algún artículo con sus firmas. Como una empresa particular, la forma de financiamiento de La Voz de la Instrucción dependía del número de suscripciones con que contaba, y de ello dan cuenta las peticiones a suscriptores y corresponsales para remitir el importe del periódico (Castilla, 1871: 43), señalando explícitamente que carecía de otros apoyos económicos y que su existencia dependía de los sacrificios, constancia y decidido empeño de su autor (Castilla, 1871: 45).

Una característica de este tipo de publicaciones es que estaban dirigidas a los maestros, lo que se confirma en el tratamiento de "comprofesor", entre el editor y los lectores en el intercambio de correspondencia.

El contenido de la publicación en cuestión está dividida en tres secciones: la oficial, la facultativa y la de anuncios, según el orden impuesto por el maestro Castilla. En la sección oficial encontramos la Ley Orgánica de la Instrucción Pública en el Distrito Federal del 15 de mayo de 1869, el Reglamento de la Ley Orgánica de Instrucción Pública en el Distrito Federal del 9 noviembre de 1869, la Ley de Instrucción Primaria en el Estado de Tlaxcala del 4 de mayo de 1868 y el Reglamento de la Ley de Instrucción Primaria en el Estado de Tlaxcala del 18 de mayo de 1868 y el Reglamento de Estudios de Puebla del 17 de junio de 1867.

La sección facultativa expone los sistemas generales y métodos especiales de enseñanza; en ella explica el sistema simultáneo, el mutuo y el mixto, lo cual, como mencionamos con anterioridad, tendría el objeto de ofrecer los elementos necesarios para elegir el mejor sistema a utilizar de acuerdo con las condiciones del establecimiento y al número de escolares y con ello obtener los mejores resultados en la enseñanza. A manera de introducción, Castilla 
refiere el mal estado en que se encontraba la educación debido a la precaria preparación de los maestros y al uso del sistema individual de enseñanza, lo que provocaba gran lentitud en el desarrollo de la actividad educativa, puesto que en una escuela con cuarenta alumnos y un total de seis horas de trabajo, cada uno de ellos aprovecharían tan sólo 4.5 minutos de lectura, 3 de escritura y 1.5 de aritmética.

En el apartado que titula "Métodos especiales de enseñanza" aborda propiamente los modelos conocidos en la época para transmitir los conocimientos en el salón de clase, esto es, las formas para enseñar la lectura, la escritura, la aritmética y la gramática cuya aplicación dependería del sistema que decidiera emplear cada escuela de acuerdo con sus recursos materiales y el número de alumnos. Conceptualiza estos métodos como los medios para inquirir la verdad, es decir, como el camino que conduce a realizar una cosa útil y conforme a un fin determinado, y resaltó su importancia pues opinaba que:

la enseñanza no puede realizarse, sin apelar a uno o algunos de ellos. Más para enseñar, es preciso decir, hablar, interrogar, preguntar, responder alternativamente, narrar y ejecutar. Cuando solamente se habla, el método se llama acromático o recitativo; si se enseña interrogando erotemático o interrogativo; si se enseña preguntando y respondiendo como interlocutor, el método se llama catequístico o interlocutivo; si se obliga a referir un párrafo o capítulo de una materia larga, aprendida de antemano, entonces se nombra narrativo; si a la referencia se acompaña el análisis y los razonamientos propios, el método es racional y por último, si la enseñanza es práctica o intuitiva, el método puede llamarse natural (Castilla, 1871: 122-123).

Para Castilla los "sistemas generales" y los "métodos especiales de enseñanza" posibilitarían la eficiente utilización de los recursos y el orden en la escuela; de manera general, los consideraba un conjunto de conocimientos a los que se les daba el nombre de "pedagogía", los cuales eran necesarios para obtener los mejores resultados en la enseñanza. Llegado a este punto, nos da una definición de lo que debía entenderse por tal práctica y nos dice: "la pedagogía trata dos asuntos: $1^{\circ}$. Los sistemas y los métodos; $2^{\circ}$. La función destinada al profesor en todas sus relaciones con los niños, con sus padres y con la sociedad" (Castilla, 1871: 123).

Esta idea que esboza el carácter de un saber sobre la educación nos permite reconocer la necesidad que ya en 1871 se tenía en México de organizar una práctica escolar desde el mismo espacio en donde ésta se daba. En cuanto a la segunda parte de su definición, sobre la función del maestro, nos deja entrever su preocupación por revalorar el trabajo del maestro y la visión que de él tenía la sociedad.

Al final de la sección facultativa toca el tema de la escuela normal señalando su importancia, su organización y los métodos que deberían utilizarse en la formación de los maestros, los que desde su punto de vista no diferían de los utilizados en la educación básica. En la 
escuela normal, la supervisión tenía que ser una actividad importante y para efectuarla propone que se verificara una vigilancia de tipo administrativa y otra facultativa. Esta última es una de sus ideas más importantes, pues la supervisión era para él la manera más adecuada para alcanzar los objetivos de la educación, por lo que esta actividad debía observarse indiscutiblemente en la formación de los profesores. Así, presenta una amplia disertación sobre el modo en que la inspección tendría que verificarse.

Propone que este tipo de escuelas se deberían subdividir en elemental y superior, formando así cuatro tipos de maestros: de elemental incompleta, de elemental completa, de instrucción superior y de instrucción normal preparatoria. Consideró que la formación del normalista tenía que darse en las mejores condiciones posibles, por lo que proponía que los alumnos deberían realizar sus estudios en calidad de pensionados.

Contra lo que se puede interpretar por su nombre, la sección de anuncios toca toda una gama de opiniones en torno a la educación; aborda de manera más libre su opinión sobre la importancia de la inspección facultativa y considera que la mejor manera de difundir la instrucción era por medio de la apertura de muchas escuelas. Es importante señalar que los lectores también opinaban sobre el tema escolar y hacían notar la falta de obras didácticas y de buenas escuelas normales.

En el número 24 de la publicación se anunciaba la finalización del primer tomo y la próxima entrega del segundo, para que los profesores siguieran contando con la información necesaria para desempeñar dignamente su noble ocupación, pero al parecer esa segunda parte nunca se realizó.

\section{México Intelectual}

Esta publicación aparece entre los años de 1889 hasta 1904, su periodicidad fue mensual inicialmente y quincenal a partir del segundo semestre de 1889; fue fundada por Enrique Conrado Rébsamen² profesor suizo que llegó a México en 1883; cabe señalar que la portada del impreso consigna como redactores propietarios, además de Rébsamen, a Emilio Fuentes y Betancourt ${ }^{3}$ y a Hugo Toff. ${ }^{4}$

\footnotetext{
2 Enrique Conrado Rébsamen, profesor originario de Suiza, al llegar a México se estableció en León, Guanajuato. En 1885 colaboró con Enrique Laubcher en la Escuela Modelo de Orizaba; fundó la Normal de Jalapa, y en 1889, la revista México Intelectual. En 1901 fue nombrado director de educación normal en la ciudad de México. Fue autor de un método de lecto-escritura basado en el uso de palabras "normales".

${ }^{3}$ Emilio Fuentes y Betancourt fue un profesor de origen cubano con grado de doctor en filosofía. Se sabe que fue sacerdote de ideas liberales y que impartió clases en escuelas metodistas en la ciudad de México. Colaborador de Rébsamen en la Normal de Jalapa, de la cual fue director entre los años de 1904 y 1909; fue redactor de la revista México Intelectual.

${ }^{4}$ Hugo Toff fue originario de Alemania, al llegar a México se estableció en Puebla. Al trasladarse a Jalapa se convirtió en colaborador de Rébsamen y de la revista México Intelectual.
} 
La redacción y administración de la revista se encontraba en la Escuela Normal de Jalapa y, debido a que la mayoría de sus colaboradores estaban vinculados a esta institución, se le ha considerado como reflejo del pensamiento y de la vida normalista de Veracruz, aunque no fue precisamente un órgano de aquélla (Zilli, 1961: 42).

Al título seguía un subtítulo que, como en el caso anterior, también refleja el objetivo del impreso: Revista pedagógica y científica literaria. En cuanto a la pedagogía, se proponía la:

difusión de los métodos y sistemas sobre los que están constituidos los establecimientos de instrucción de los pueblos más civilizados, se proponen, no sólo exponer teóricamente cuanto tenga de mediata o inmediata relación con los tales procedimientos de la Pedagogía, sino, a la vez, presentar a sus compañeros en el magisterio, dispersos por el vasto territorio nacional, una guía práctica que les indique, satisfactoriamente, y sin que tengan que desplegar grandes esfuerzos, la aplicación acertada de las modernas enseñanzas pedagógicas (México Intelectual, 1889: 2).

Además, tenía la intención de propagar cuanto movimiento científico y literario se diera en el territorio nacional, asimismo, se deslindaba de cualquier interés ajeno a la difusión de la educación y hacía expresa referencia a no querer entrar en polémicas de ningún tipo.

La publicación se editó desde 1889 y hasta 1901 en Jalapa, año en que Rébsamen fue nombrado director de la Normal de la ciudad de México, por lo que ésta tuvo que suspenderse debido a que las labores de reorganización de la escuela absorbieron todas las energías del maestro. En 1903 reanudó su publicación en la capital del país, bajo nueva dirección y con mayor dimensión (Zollinger, 1935); finalmente se suspendió en 1904, año de la muerte de su fundador.

La revista tuvo un formato de $25 \times 17$ cm y cada número constó de 56 páginas con un costo de suscripción de 50 centavos en su etapa mensual y de 25 en su aparición quincenal con sólo 32 folios. Lamentablemente no se consigna el número de su tiraje y solamente de manera muy superficial su alcance geográfico al señalar que se contaban con agentes en Veracruz, Chicontepec, Puebla, Orizaba, Cosamaloapan, Tlacotálpam y México. Sin embargo, el hecho de que avisara que quienes desearan adquirirla y habitaran en lugares en donde no hubiera agentes remitieran diez centavos como importe de timbres, nos hace suponer que llegó a otros lugares diferentes a los señalados anteriormente.

Otro indicio de sus posibles alcances nos lo ofrecen las cartas, remitidos y reproducciones recibidos por la redacción y provenientes de diferentes lugares, no sólo de la República Mexicana, sino de otros países como Guatemala, Uruguay, Argentina, Perú y Cuba, en los que se dan noticias del estado de la educación en estos lugares y del congreso educativo en Chile en 1890 (México Intelectual, 1890: 120). 
La relación de la publicación con personalidades educativas extranjeras se puede explicar debido a que Rébsamen era miembro y representante en México del Consejo Permanente e Internacional de Educación, órgano al cual promovió en México Intelectual. Esta asociación tenía como fin la unificación internacional de los principios pedagógicos por medio de la discusión de temas educativos cuyos resultados publicaría en su órgano de difusión llamado Diario de Correspondencia, impreso en alemán, francés y español. Entre los miembros de esta asociación se podían contar inspectores escolares, ministros del ramo de educación, secretarios de agrupaciones educativas, directores de escuelas normales y de escuelas municipales, profesores y editores de revistas pedagógicas (México Intelectual, 1890: 149-154).

Entre los colaboradores de la revista veracruzana se mencionan a Ignacio Manuel Altamirano, catedrático de la Escuela Normal de México; a los profesores de la Escuela Normal de Jalapa, Juan Manuel Betancourt y Manuel R. Gutiérrez; a José Miguel Macías, maestro y vicerrector del Colegio Preparatorio de Veracruz; a Vicente Mora, inspector de instrucción pública de escuelas en el estado de Sonora; a Rodolfo Menéndez, inspector de escuelas en el estado de Yucatán; a Esteban Morales, catedrático y rector del Colegio Preparatorio de Veracruz; a Luis E. Ruiz, docente en la Escuela Normal de México y profesor adjunto de la de Medicina; y por último, a José María Vigil, catedrático de la Escuela Nacional Preparatoria y director de la Biblioteca Nacional.

Colabora también Rafael de Zayas Enríquez, quien fuera representante de Veracruz en la Exposición de París de 1889 (México Intelectual, 1890: 224). De igual modo, encontramos artículos de Dolores Correa Zapata, Porfirio Parra, Graciano Valenzuela, Federico Sandrock y traducciones de artículos de Gabriel Compayré, entre otros. En fin, la revista vinculaba a una parte significativa de la élite educativa de su tiempo.

Constantemente se hace referencia al pago de las suscripciones, por lo que suponemos que su financiamiento, o parte del mismo, dependía de ellas, sin embargo, Martha Curiel considera que recibía el respaldo económico de Juan de la Luz Enríquez, gobernador de Veracruz (1884-1892), el que se suspendió a la muerte del gobernante, pero gracias a la importancia de la publicación pudo seguir editándose con los ingresos de las suscripciones (Curiel, et al., 1988). Otra fuente de financiamiento, aunque tal vez marginal, fueron las inserciones pagadas, pues la publicación mencionaba que todo aquel interesado en anunciarse en sus páginas lo podría hacer a "precios convencionales".

El contenido de México Intelectual fue muy variado pero principalmente se enfocó a la difusión de los métodos pedagógicos en uso. Así, encontramos artículos sobre el mejor método para el aprendizaje de la lectura, lecciones sobre geometría, la enseñanza de la caligrafía en la escuela primaria, metodología aplicada a la enseñanza de la historia, de la geografía o de las ciencias naturales en la escuela primaria. También ofrece algunas noticias de lo que sucedía en otras partes del continente, como cuando explica los métodos de lectura y del canto en Uruguay. 
Los contenidos difundidos en esta publicación nos ubican en una época en que se vivía la modernización de las técnicas de enseñanza y en la que se observa que el sistema que se cree adecuado utilizar era el simultáneo. Empero, dos son las temáticas fundamentales abordadas en la revista, la primera giraba en torno a un saber pedagógico, y la segunda, alrededor de las formas en que el conocimiento debía transmitirse en el salón de clases, es decir, en torno a la didáctica.

En un plano estrictamente teórico ubicamos artículos sobre el sistema de Froebel, sobre "pedagogía moderna" escritos por Rébsamen y la disertación de Porfirio Parra sobre la definición y progresos de esta actividad. La mención de estas dos colaboraciones no es casual, pues muestra que la publicación estaba abierta a todas las opiniones, ya que debe resaltarse que ambos escritores representaron dos posiciones pedagógicas diferentes: la liberal y la positivista.

Respecto al subtítulo del periódico, que menciona la ciencia y la literatura, encontramos artículos sobre la cultura popular, sobre las características distintivas de la literatura nacional y la reflexión sobre si en México existía una que mereciera tal denominación. En el campo de la ciencia se menciona al telégrafo, al telescopio, a la geología, a la paleontología y a la arqueología, entre otros temas.

La primera parte de cada número se aboca a reproducir los artículos de los colaboradores y, en la parte final, aparecen dos secciones, "Boletín Bibliográfico" y "Variedades". La primera es un rico mosaico de obras sobre educación, en la que se daban noticias sobre las publicaciones en canje que recibía la redacción. También se hacían breves reseñas de libros, entre los que podemos mencionar: La moneda de Emilio Rabasa, el Diccionario histórico y bibliográfico de García Cubas, algunos textos utilizados en aquellos años en la primaria, como el Primer Libro de Lectura y Aritmética Elemental de Luis E. Ruiz o la Geografía de México de Alberto Correa.

La relación con otros países también está presente en esta sección, así, podemos observar que se recibían el Journal de Correspondance de la junta provisional para la instalación de un Consejo Permanente e Internacional de Educación, y Erziehungs-Blätter, órgano de la Asociación Alemana-Americana de Maestros.

La sección de "Variedades", correspondiendo a su nombre, exponía un sin número de noticias referentes a planteles educativos, inventos, memorias sobre instrucción de los estados de la república y de algunos otros países, reseñas de festividades y de periodos de exámenes en algunos establecimientos escolares, así como información de actividades de personajes relacionados con la educación. De igual manera, encontramos referencias al Congreso de Instrucción de 1890.

Detenernos en los artículos de esta publicación nos da oportunidad de reconocer cómo sus contenidos reflejan los cambios que fue teniendo la visión sobre la educación con el paso 
del tiempo. Por ejemplo, en contraste con lo que opinaba Castilla en 1871 sobre que la manera más eficiente de difundir la instrucción estaba en la apertura del mayor número de establecimientos educativos, hacia 1889, México Intelectual planteaba una visión diferente al señalar que:

\begin{abstract}
lo que importa no es tener muchas escuelas, sino pocas, pero bien organizadas. El gran principio económico de la división del trabajo tiene su aplicación en la escuela lo mismo que en la industria. La no aplicación de este principio es la causa principal del estado verdaderamente lamentable que guardan las escuelas municipales de la capital (México Intelectual 1889: 213).
\end{abstract}

La muerte de Rébsamen en 1904 motivó el fin de la publicación y el inicio de una nueva redactada por sus discípulos, México Pedagógico. Algunos autores como Martha Curiel (1988) advierten, con la aparición del nuevo impreso, una abierta confrontación entre los seguidores de Rébsamen y los de Torres Quintero. Otros, como René Avilés (1967), sólo vieron en la naciente publicación la continuación de México Intelectual en los años 1904-1905, bajo la dirección de Luis J. Jiménez.

La observación de una confrontación entre los partidarios de los mentores citados, se puede considerar como el inicio del debate que sostuvieron ambos grupos para demostrar que el método propuesto por sus respectivos maestros era el más adecuado para la enseñanza de la lectura y escritura. La controversia fue ventilada a través de las páginas de México Pedagógico y de La Enseñanza Primaria, periódico fundado algunos años antes por Torres Quintero.

\title{
La Enseñanza Primaria
}

Consideramos La Voz de la Instrucción como el esfuerzo personal de Antonio P. Castilla, en cambio, México Intelectual fue el trabajo de un grupo de educadores aglutinados en torno a un personaje de gran trascendencia como fue Enrique C. Rébsamen, quienes hasta cierto punto contaron con el apoyo de una institución como fue la Normal de Jalapa.

La Enseñanza Primaria. Quincenal Pedagógico, guarda cierta similitud con México Intelectual, pues es producto de la iniciativa de una agrupación: "Colegio de Profesores Normalistas de México". Observamos, por tanto, que las publicaciones pedagógicas pasaron de ser iniciativas individuales de maestros a convertirse en impresos orgánicos de asociaciones de profesores, e incluso, de las instituciones que se fueron creando con los cambios que iban conformando el sistema educativo.

Ejemplo de esto último es La Escuela Mexicana que fue editada en 1904 por la Dirección General de Instrucción Primaria del Distrito y Territorios Federales, o la Revista de la Instrucción 
Pública de 1896, que fue sustituida en 1903 por el Boletín de la Instrucción Pública Mexicana, órgano de la Secretaría de Instrucción Pública. El fin que se perseguía ya no sólo respondía a la preocupación por unificar la forma de transmisión del conocimiento, sino que se pretendía uniformar los criterios administrativos en la educación primaria, lo que muestra que las publicaciones pedagógicas reflejan el desarrollo del cada vez más complejo sistema educativo mexicano.

La Enseñanza Primaria (julio 1901: 2) tenía la intención de abordar aquellos asuntos que tuvieran relación con la práctica metodológica en ese nivel y los que se vincularan con la legislación escolar, señalando algunas deficiencias administrativas. Su fundador y director fue Gregorio Torres Quintero (1866-1934)5 y Celso Pineda, ${ }^{6}$ su editor; además fungieron como socios fundadores Luis de la Brena ${ }^{7}$ y Ponciano Rodríguez. ${ }^{8}$

La publicación en pequeño formato de $23 \times 16 \mathrm{~cm}$, con periodicidad quincenal, apareció los días 1 y 15 del mes con un costo de 0.65 pesos la suscripción semestral y de 1.25 pesos anuales, pagados por adelantado. Adicionalmente debían de pagarse 0.25 pesos por concepto de gastos de giro. Constaba de 16 páginas, en las que colaboraban Gregorio Torres Quintero, Ángel Pola, Daniel Delgadillo, Jesús Sánchez, Justo Sierra, Ponciano Rodríguez, Rodolfo Menéndez, entre otros. Asimismo contaba con reproducciones de artículos de Carlos A. Carrillo, Vicente Riva Palacio y Juan de Dios Peza.

${ }^{5}$ Gregorio Torres Quintero fue un profesor nacido en Colima; en marzo de 1883 obtuvo el título de "Preceptor de Primer Orden", en el Liceo de Varones de su estado natal, en donde ejerció su profesión hasta 1887; al año siguiente fue pensionado por el gobierno del estado para estudiar en la Escuela Normal de Profesores de la ciudad de México, en la que se graduó en 1891. De regreso a Colima fundó una Escuela Modelo y fue nombrado jefe del ramo educativo, realizando con éxito lo que se conoció como "La Reforma Escolar Colimense". Por sus méritos fue llamado por la Dirección General de Instrucción Primaria del Distrito Federal y Territorios en donde, entre 1898 y 1904, fue oficial segundo, oficial primero y secretario general. En 1904 fue nombrado por Justo Sierra jefe de la Sección de Instrucción Primaria y Normal. En abril de 1917 fue enviado por Carranza a Yucatán para hacerse cargo del ramo de educación; de regreso a México en 1918, se dedicó a la redacción de libros escolares y a la creación de la Secretaría de Educación Pública, fue nombrado Consejero Técnico, se jubiló en 1923.

${ }^{6}$ Celso Pineda originario de Taxco Guerrero, estudió en la Normal de la capital de la República de la cual fue su director. Maestro de enseñanza elemental, director de primaria e inspector escolar, también escribió textos que fueron usados durante muchos años en la escuela primaria.

${ }^{7}$ Originario de la ciudad de México, Luis de la Brena fue egresado de la Normal capitalina. Profesor de profesión, también fue director de primaria e inspector escolar, profesor y director de la escuela Normal de la ciudad de México.

8 Ponciano Rodríguez era originario de Chimalhuacán, municipio de Ozumba en el Estado de México. En 1889 ingresó a la Escuela Normal de la Ciudad de México en donde, tiempo después, fue profesor de la Primaria Anexa. En 1895 fue miembro de la Comisión Dictaminadora de Libros de Texto, siendo el primer maestro de primaria que formó parte de una de ellas. Hacia 1907 la secretaría del ramo nombró una comisión para revisar las leyes, reglamentos y programas de enseñanza primaria siendo él el único profesor considerado. Entre otros cargos fue inspector pedagógico de las escuelas primarias del Distrito Federal, fundador y director de la Escuela Normal Nocturna, director de la Primaria Anexa, jefe de Instrucción Rudimentaria en sustitución de Torres Quintero y secretario de la malograda Asociación Nacional del Magisterio, director del periódico La Evolución Escolar, miembro de la Sociedad Científica Antonio Alzate, entre otros cargos. 
Con base en las obras que se remitían a la redacción, además del correo y la mención de la localización de sus agentes, podemos saber que La Enseñanza Primaria circuló en Mihauatlán y Etla, Oaxaca; Las Esperanzas, Coahuila; Hermosillo, Álamo y Caborca, Sonora; San Dimas, Durango; Guanajuato, Guanajuato; Chilpancingo, Guerrero; Temascalapa y Mezquetitlán, Hidalgo; Lagos de Moreno, Jalisco; Amanalco de Becerra, México; Valle de Allende, Nuevo León; Puebla e Izúcar de Matamoros, Puebla; Querétaro, Querétaro; San Luis Potosí, S.L.P.; Tampico, Tamaulipas; Tepic, Nayarit y Panotla, Tlaxcala (La Enseñanza Primaria, 1901: 80).

De los pocos datos que se consignan podemos inferir que su financiamiento provenía del pago de las suscripciones e incluso, para evitar mayores gastos a los lectores del Distrito Federal, se les pedía que acudieran directamente a hacer su pago con Celso Pineda para evitar el recargo de 0.25 pesos por concepto de giro (La Enseñanza Primaria, 1905: 187). La publicación abordó tópicos sobre geografía, aritmética, lengua nacional, lecciones de cosas, método de lectura, instrucción cívica, historia, física, gramática, higiene, historia de la educación, moral, libros de texto, exámenes, avances científicos, entre otros.

Un tema recurrente en este impreso es el de la crítica a la utilización del libro de texto, lo cual muestra la influencia ejercida por Carlos A. Carrillo entre los colaboradores de la publicación. Por ejemplo, Luis de la Brena decía que "la enseñanza predominantemente libresca que por desgracia aún no se ha desterrado [...] preside por regla general todos los actos escolares, las lecciones de cosas no han escapado a prácticas tan viciosas" (La Enseñanza Primaria, 1901: 10). Una opinión más moderada sobre la utilización de textos en la escuela primaria la encontramos en las palabras de Ponciano Rodríguez, quien afirmaba que no creía que "el libro sea el alma de la escuela, pero sí que [era] un auxiliar poderoso para el maestro y un elemento importante [...] para la cultura intelectual y ulterior del alumno" ( $\mathrm{La}$ Enseñanza Primaria, 1901: 78).

Asimismo, esta publicación aborda el tema de la enseñanza de la lectura; Celso Pineda cuestionaba que la práctica de la lectura resintiera la supresión de los libros de texto, lo que le parecía erróneo pues para él la verdadera causa de que no se llegara a leer bien no era lo poco que se practicaba la lectura, sino el mal procedimiento que se seguía para enseñarla ( $L a$ Enseñanza Primaria, 1901: 50).

Durante mucho tiempo la forma en que se enseñaba a leer y escribir fue una preocupación presente en los pedagogos de fines del siglo XIX, un primer paso hacia la resolución de este problema fue la simultaneidad en su enseñanza. Al respecto, Rébsamen propuso el método simultáneo y basado en las "palabras normales" que consistía en utilizar vocablos cortos que contuvieran todos los tipos de sílabas y letras del alfabeto. En esta revista podemos encontrar indicios del debate con un tono marcadamente pedagógico en torno a la mejor manera de enseñar a leer y escribir, ejemplo de ello son varios artículos que mencionan algunas inconsistencias en la aplicación del método de Rébsamen, entre las que se señalaba 
la utilización de palabras con irregularidades ortográficas como kiosco o wagón. No obstante, esta metodología había sido reconocida como la adecuada para la enseñanza simultánea de la lectura y escritura por la Ley Reglamentaria de Instrucción Primaria Obligatoria de 1891 (La Enseñanza Primaria, 1901: 34).

A mi juicio, dicha consideración era una incipiente crítica que fue preparando el camino para la posterior aparición del método onomatopéyico de Torres Quintero en 1904, manual clásico en la enseñanza de la lectura-escritura del editor de La Enseñanza Primaria.

Para 1901, año del inicio de esta publicación, la posición teórica de su fundador le hizo advertir que "la actividad pedagógica de un pueblo no sólo se manifiesta en sus doctrinas y teorías, sino en sus métodos e instituciones prácticas" (La Enseñanza Primaria, 1901: 3). Por tanto, podemos pensar que para Torres Quintero se había llegado a una etapa de formalización de un saber pedagógico que a su vez había permitido el desarrollo de la didáctica y la apertura de establecimientos en donde se podían aplicar estos progresos, es decir, una escuela en donde no sólo se proporcionara instrucción, sino que ofreciera una educación basada en los principios pedagógicos construidos por los avances teóricos de fines del siglo XIX, los que se fueron adicionando a las diferentes leyes de la época. Insistimos que en este proceso educativo es claro el papel que jugaron las publicaciones pedagógicas, pues fueron el medio de difusión de las ideas reformadoras de la educación.

En este orden de ideas, en La Enseñanza Primaria se señalaba que en la escuela se debía privilegiar las "lecciones de cosas" al decirse que "i[...] bastaría esta sola asignatura para realizar esa concepción maravillosa que debe perseguirse como fin único y fundamental de la buena escuela: enseñar a pensar!” (La Enseñanza Primaria, 1910: 10). Esta visión no olvida la importancia del ejercicio físico como medio para evitar el cansancio intelectual pues su adecuada aplicación proporcionaba descanso al cerebro y vigor al cuerpo (La Enseñanza Primaria, 1901: 5).

La formación del ciudadano y el desplazamiento de la antigua fidelidad a la Iglesia en favor de la Nación se hace presente en este impreso por medio de una lección sobre instrucción cívica en la que Torres Quintero, a cuestionamiento de un alumno que oyó decir al cura que el gobierno hacia mal en forzar la compra de timbres, explicó que el gobierno utilizaba ese dinero para el financiamiento de servicios y la creación de instituciones y que si todos pensaran como el cura, "no habría nada, es decir, no habría gobierno, y ya hemos dicho que sin él no puede existir una sociedad" (La Enseñanza Primaria, 1901: 64).

Un tema que bien pudiéramos considerar como un antecedente temprano de orientación vocacional es el que aborda Luis de la Brena, al considerar que si para subsistir es inevitable ejercer una profesión u otra actividad cotidiana, es necesario reflexionar sobre cuáles son las inclinaciones y aptitudes del individuo antes de que éste elija alguna, pues de la elección correcta dependerá su porvenir (La Enseñanza Primaria, 1901: 40). 
En 1913 Torres Quintero fue nombrado jefe de instrucción rudimentaria, tema en el que no era un improvisado, pues desde 1901 expresó su preocupación por este tipo de educación. Consideraba que las escuelas urbanas eran las únicas atendidas en tanto las rurales estaban en el olvido. Reflexionaba que "mientras las escuelas rurales sigan en el abandono [...] la mayoría de los mexicanos seguirá sumida en las tenebrosidades del no ser" (La Enseñanza Primaria, 1901: 128).

En el panorama que este pedagogo nos presenta se aprecia que, a pesar de la intención de hacer llegar la educación hasta los lugares más apartados, existía un cierto descuido en la organización y apoyo a la enseñanza de las escuelas alejadas de los centro de población. Es decir, los avances educativos y el soporte económico oficial eran evidentes en las escuelas urbanas en contraste con las de tipo rural, por lo que, en consecuencia, se fue abriendo una brecha profunda en la calidad de la educación impartida en ambos tipos de establecimientos educativos, que persiste hasta nuestros días.

Se pensó que La Enseñanza Primaria se suspendería el $1^{\circ}$ de julio de 1911, sin embargo, la publicación continuó e incorporó un mensaje de Celso Pineda en el que exponía que debido a ciertos problemas, que no especificaba, estuvieron a punto de concluir su labor periodística. No obstante, los siguientes números carecen de portada, lo que indica que si bien sus editores pudieron sortear ciertas dificultades era evidente el final de este impreso, que ocurrió en los últimos días de 1911.

A manera de conclusión, considero necesario reconocer a la prensa pedagógica como un conjunto de impresos con características propias, entre las que estaban no sólo la difusión de nuevos saberes instructivos. Ha de reconocérsele el carácter de medio formador de maestros que algunos profesores, en su calidad de intelectuales pedagógicos, utilizaron con la intención de que los mentores empíricos y todo aquel que tuviera a su cargo un establecimiento escolar, tuviera acceso a los conocimientos básicos de la incipiente práctica pedagógica que se fue desarrollando a lo largo de la segunda mitad del siglo XIX en México.

Distinguir y apreciar su carácter de verdaderos tratados teóricos sobre educación es el rasgo distintivo que los distancia de la categoría de publicaciones educativas o infantiles, como se les ha clasificado y que señalé líneas arriba. Este atributo es el que se debe considerar para revalorar su importancia como fuente para historiar la educación, pues éstas deben tomarse como testimonio de la evolución del pensamiento pedagógico decimonónico.

Fijar la mirada en la prensa pedagógica estriba también en que sus contenidos son la huella de uno de los personajes fundamentales en la reforma educativa de la época: los maestros. Mirar el pasado educativo a través de los ojos de los profesores nos permite observar otros ángulos del devenir educativo, lo que contribuye a contar con los elementos necesarios para la reconstrucción, no solamente del pensamiento educativo, sino también de la práctica pedagógica en nuestro país. 
El estudio de las publicaciones periódicas pedagógicas nos lleva al encuentro de las argumentaciones técnicas de las reformas escolares, de la influencia de pedagogos extranjeros en la construcción del saber educativo nacional y al encuentro de los indicios que revelen cómo, los maestros normalistas se fueron conformando en una élite intelectual que no fue ajena a confrontaciones grupales por detentar la autoridad, tanto pedagógica como de poder, en las instituciones creadas por la estructuración del sistema educativo mexicano.

\section{Fuentes consultadas}

\section{Bibliografía}

Abramo, Marcelo y Yolanda Barberena (1998), El estadio. La prensa en México (1870-1879), México, Instituto Nacional de Antropología e Historia.

Alvarado, María de Lourdes (2004), La educación superior femenina en el México del siglo xIx. Demanda social y reto gubernamental, México, Universidad Nacional Autónoma de México-Plaza y Valdés.

Alvear, Carlos (1999), Breve historia del periodismo, México, Jus.

Avilés Fabila, René (1967), Enrique C. Rébsamen. Quetzalcóatl de la Educación, México, Secretaría de Educación Pública (Cuadernos de Lectura Popular).

Becerril, Elizabeth (2000), "Condición y educación de la mujer mexicana durante la primera mitad del siglo XIX a través de la prensa", tesis de licenciatura, Facultad de Filosofía y Letras, Universidad Nacional Autónoma de México, México.

Bringas, Guillermina y David Mascareño, (1988) Esbozo histórico de la prensa obrera en México. México, Instituto de Investigaciones Bibliográficas/Universidad Nacional Autónoma de México.

Castellanos, Abrham (1909), Pedagogía Rébsamen. Asuntos de metodología general relacionados con la escuela primaria y recopiladas por el profesor Abraham Castellanos, México, Librería de Ch. Bouret.

Castilla, Antonio (1871), La Voz de la Instrucción, o sea el libro primero del maestro. Semanario destinado al progreso de la enseñanza y a la defensa de los intereses materiales y morales del profesorado mexicano, México, Imprenta y Librería de la Enciclopedia de Instrucción Primaria.

Cervantes, Manuel (1874), El Educador Mexicano, 21 de agosto de 1874.

Civera, Alicia (coord.) (2002), Debates y desafíos en la historia de la educación en México, México, El Colegio Mexiquense, A. C.

Covo, Jacqueline (1993), "La prensa en la historiografía mexicana" en Historia Mexicana, vol. 42, núm. 3 (167), enero-marzo, pp. 689-710.

Curiel, Martha, et al. (1998), Rébsamen y la Revista México Intelectual, México, Universidad Pedagógica Nacional (Colección Educadores Mexicanos 1).

Galván, Luz Elena (2004), "Creación del ciudadano: los intelectuales y la prensa infantil, 1870-1900", Historia y Grafía, núm. 23, pp. 217-262.

Larroyo, Francisco (1973), Historia Comparada de la Educación en México, México, Porrúa.

Lombardo, Irma (1992), De la opinión a la noticia. El surgimiento de los géneros informativos en México, México, Kiosco.

Martínez Moctezuma, Lucía (2004), "Retrato de una élite. Autores de libros escolares en México (1890-1920)", en Carmen Castañeda (coord.), Lecturas y lectores en la historia de México, México, CIESAS-El Colegio de Michoacán-Universidad Autónoma del Estado de Morelos. 
Meneses, Ernesto (1998), Tendencias oficiales educativas oficiales en México 1821-1911, México, Universidad Iberoamericana.

Ochoa, Moisés (1968), Reseña histórica del periodismo mexicano, México. Porrúa.

Pereda, Alicia (2003), "De las 'damas melindrosas' a las 'señoritas amables': las mujeres en la prensa mexicana a comienzos del siglo XIX", Cuadernos americanos, año XVII, vol. 4, núm. 100, julio-agosto, pp. 160-180.

Ruiz Castañeda, María del Carmen, Luis Reed y Enrique Cordero (1974), El Periodismo en México. 450 años de historia.,México, Editorial Transición. - Irma Lombardo y María Teresa Camarillo (1984), La prensa en México siglo xIx. Exposición gráfica, México, Instituto de Investigaciones Bibliográficas/Hemeroteca Nacional de México/Universidad Nacional Autónoma de México.

Salas, Consolación (1998), "Las revistas masculinas mexicanas a principios del siglo Xx", en Laura Navarrete y Blanca Aguilar (coords.), La prensa en México. Momentos y figuras relevantes (1800-1915), México, Addison Wesley Longman.

Toussaint, Florance (1989), Escenarios de la prensa en el porfiriato, Colima, Fundación Manuel Buendía/ Universidad de Colima.

Vergés, Josep María Miquel i (1985) [1941], La independencia mexicana y la prensa insurgente, (edición facsimilar), México, Instituto Nacional de Estudios Históricos de la Revolución Mexicana-Comisión Nacional para las Celebraciones del 175 de la Independencia Nacional y 75 Aniversario de la Revolución Mexicana.

Wold, Ruth (1970), El Diario de México: primer cotidiano de Nueva España, Madrid, Gredos.

Zilli, Juan (1961), Historia de la Escuela Normal Veracruzana, México, Citlaltepetl (Suma Veracruzana. Historiografía).

Zollinger, Edwin (1935), Enrique C. Rébsamen el renovador de la instrucción primaria, México, Secretaría de Educación Pública.

\section{Hemerografía}

Boletín Bibliográfico y Escolar (1891), 16 de mayo de 1891.

Enseñanza Moderna, La, (1897) $1^{\circ}$ de octubre de 1897.

Enseñanza Objetiva, La, (1879) 4 de enero de 1879.

Enseñanza Primaria, La, (1901) 1º de julio de 1901.

Hijas del Anáhuac, Las, (1887) 4 de diciembre de 1887.

México Intelectual, (1889) enero-junio de 1889.

Porvenir de la niñez, El, (1870) 16 de diciembre de 1870.

Juan Pedro Galván Gómez. Facultad de Filosofía y Letras, UNAM. Estudiante de Maestría en Historia. Línea de investigación: El discurso pedagógico de los maestros normalistas a principios del siglo Xx. Ganador del segundo lugar en el concurso de "La mejor tesis de licenciatura en Historia de la Educación", SEP, diciembre 2011, con la tesis "La prensa pedagógica (1871-1901). Indicios de un nuevo discurso".

Recibido: 23 de febrero de 2012

Aceptado:24 de octubre de 2012 